\title{
Using Deep Learning Neural Network for Block Partitioning in H.265/HEVC
}

\author{
Ming Yang ${ }^{1}$, Ying $\mathrm{Xie}^{1}$, Jian $\mathrm{Yu}^{2}$, Zhe Wang ${ }^{3}$, Tao $\mathrm{Wu}^{1}$ \\ \{mingyang@kennesaw.edu, yxie2@kennesaw.edu, yujian@tju.edu.cn, snowleoperd@126.com, twu6@students.kennesaw.edu\} \\ College of Computing and Software Engineering, Kennesaw State University, Marietta, GA 30060, USA ${ }^{1}$ \\ School of Computer Science and Technology, Tianjin University, Tianjin 300072, China ${ }^{2}$ \\ College of Science, Tianjin University of Technology, Tianjin 300384, China ${ }^{3}$
}

\begin{abstract}
Coding Tree Units (CTUs) and Coding Units (CUs) is a critical task of video compression in H.265/HEVC video coding standard. In this paper, we utilize deep learning techniques, especially the deep Convolutional Neural Network (CNN) to speed up the block partitioning process. Deep CNNs have achieved break-through improvements on image recognition tasks such as image classifications, object identifications, and image annotations. However, very few work has been done in applying deep CNN to video encoding. Block partitioning in video coding is highly dependent on the content of the video frames, and thus it is natural to take advantage of the significant capabilities of deep CNN on image content detection and recognition to perform block partitioning and avoid the time-consuming iterative RateDistortion-Optimization (RDO) process. Experimental results have shown that the proposed methodology has largely speed up the coding process and has also achieved coding efficiency comparable to the reference software of H.265/HEVC.
\end{abstract}

Keywords: Deep Learning, Deep CNN, H.265, HEVC, Video Encoding.

\section{Introduction}

Video coding techniques have been there for decades to enable storage and transmission of digital video contents with limited storage space and transmission bandwidth. The past video coding standards, such as H.263, MPEG-2, H.264, have adopted the hybrid coding architecture which utilized block coding, intra prediction, motion estimation, transformation, and entropy coding to achieve high level of compression efficiency. The latest video coding standard, H.265/HEVC, has inherited such type of hybrid coding architecture. It has made improvements in each of the coding modules and overall it has achieved 50\% compression performance gain compared to H.264AVC.

H.265/HEVC has adopted a more flexible blocking strategy, a more sophisticated data structure, more choices on intra-prediction modes, and other advanced techniques to achieve the above performance goal. The tradeoff is more intensive computation, which hinders its penetration to real-time streaming/transmission applications scenarios at current stage. Many coding decisions have to be made real-time during coding process, such as blocking ( $\mathrm{CTU} \rightarrow \mathrm{CU}, \mathrm{CU} \rightarrow \mathrm{PU}, \mathrm{CU} \rightarrow \mathrm{TU}$ ), prediction mode decision (intra-mode vs. inter mode), prediction direction decision in intra-prediction. 
All these decisions are dependent on the contents of the video frames and making such decisions often times require exhaustive search if Rate-Distortion Optimization (RDO) is needed. In real-time streaming, these decisions need to be made as fast as possible and thus exhaustive search is infeasible. In recent years, Convolutional Neural Network (CNN) has made great advances in the analysis and recognition of image/video contents. Thus, it is natural to apply trained CNNs to perform the above mentioned coding decision making process to largely speed up the coding process of H.265/HEVC and make it feasible for real-time coding and streaming applications. In the following sections, the proposed ideas will be discussed in further details.

\section{Background and Literature Review}

Fig. 1 has illustrated the traditional RDO-based exhaustive search CU partitioning process.
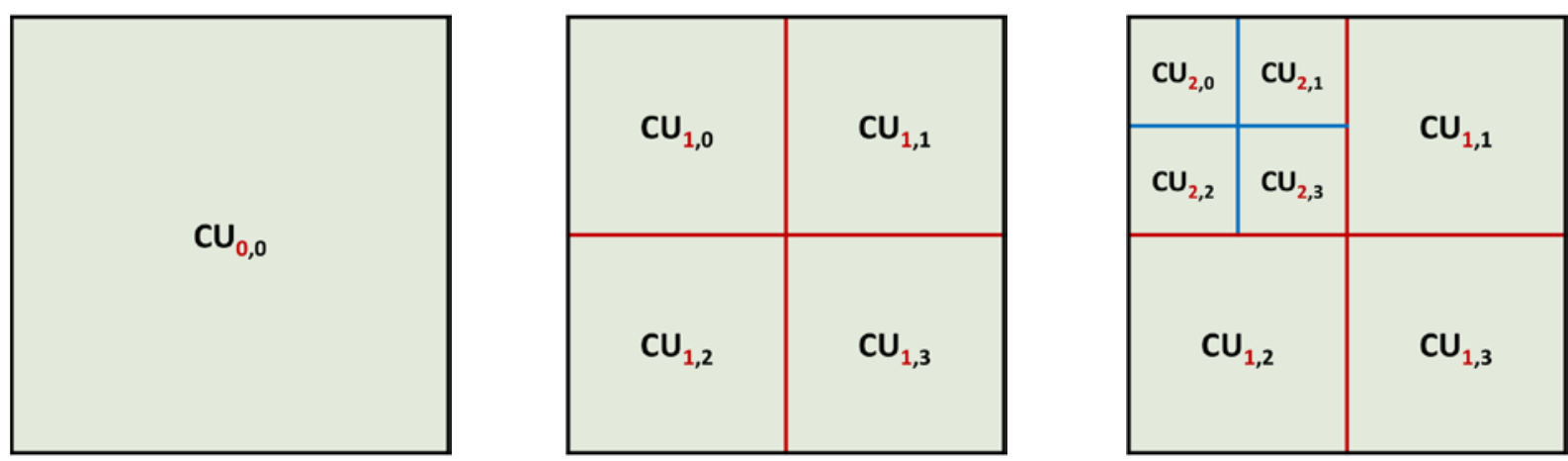

1. Suppose $\mathrm{CU}_{0,0}$ is the optimal coding mode

2. Calculate $J_{0,0}$

3. Split $\mathrm{CU}_{0,0}$ into four smaller CUs of size of $16 \times 16$ : $\mathrm{CU}_{1,0}, \mathrm{CU}_{1,1}, \mathrm{CU}_{1,2}$ and $\mathrm{CU}_{1,3}$

4. Calculate $J_{1,0}$

5. Split $\mathrm{CU}_{1,0}$ into four smaller CUs of size of $8 \times 8: \mathrm{CU}_{2,0}, \mathrm{CU}_{2,1}, \mathrm{CU}_{2,2}$ and $\mathrm{CU}_{2,3}$

6. Calculate $J_{2,0}, J_{2,1}, J_{2,2}$ and $J_{2,3}$

7. Compare $J_{1,0}$ and $\left(J_{2,0}+J_{2,1}+J_{2,2}+J_{2,3}\right)$ if $J_{1,0}>J_{2,0}+J_{2,1}+J_{2,2}+J_{2,3}$ else split $\mathrm{CU}_{1,0}$

keep $\mathrm{CU}_{1,0}$

8. Make decision on $\mathrm{CU}_{1,1}, \mathrm{CU}_{1,2}$ and $\mathrm{CU}_{1,3}$

9. Make decision on $\mathrm{CU}_{0,0}$, ( compare $J_{0,0}$ and $J_{1,0}+J_{1,1}+J_{1,2}+J_{1,3}$ )

10. Finally we get the optimal coding mode

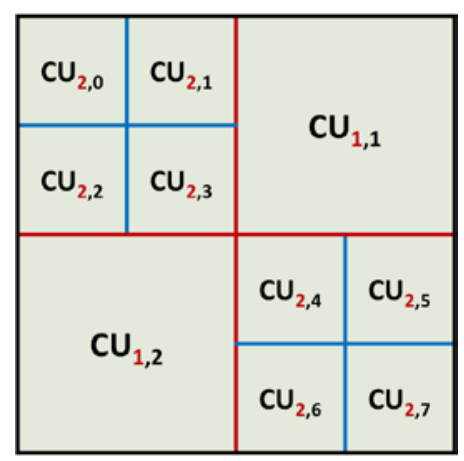

Fig. 1 Iterative Searching Process in CU Partition in H.265/HEVC Reference Software

A novel fast Coding Tree Unit partitioning for HEVC/H.265 encoder was proposed in [1]. This method does not require any pre-training and provides a high level of adaptivity to the dynamic changes in video contents; it relies on run-time trained neural networks for fast Coding Units splitting decisions. Paper [2] proposed a machine learning based approach for fast CU partition decision using features that describe CU statistics and sub-CU homogeneity. The proposed scheme was implemented as a "preprocessing" module on top of the Screen Content Coding reference software. Liu et al. ([4] [5]) devised a CNN based fast algorithm to decrease no less than two CU partition modes in each CTU for full rate-distortion optimization (RDO) processing, thereby reducing the encoder's hardware complexity. In another study, Chen et al. [6] proposed a fast coding unit (CU) depth decision algorithm for intra coding of HEVC using an artificial neural network (ANN) and a support vector machine (SVM). Machine learning provides a systematic approach for developing a fast algorithm for early CU splitting or termination to reduce intra 
coding computational complexity. Compared with existing efforts that applied machine learning in video encoding, our proposed methodology has the following two unique features: (1) it takes advantages of superiority of the-stateof-the-art deep CNN technology on image content detection to enhance content-based video encoding; (2) it utilizes deep CNN as the primary technique for multiple content-relevant tasks in video encoding within the framework of H.265/HEVC.

\section{Using CNN to Divide CTU into CUs}

Coding Tree Unit (CTU) is the basic logic unit of the H. 265/HEVC standard and replaces macroblocks that were used in the previous standards. CTUs can be 16x16, 32x32, or 64x64 pixels in size. Larger size of CTU typically improves video encoding efficiency [7][8], especially for higher-resolution video. Each CTU can be partitioned recursively into coding unit (CU). The smallest CU can be 8x8. A CTU can be one CU or partitioned into 4 equal-size $\mathrm{CU}$. Each $\mathrm{CU}$ that is larger than $8 \mathrm{x} 8$ can remain as one $\mathrm{CU}$ or can be further partitioned into 4 equal-size CU. A quadtree structure can be used to represent the partition of a CTU into CUs, as shown in Fig. 2. Given a CTU with size of 64x64, instead of recursively partitioning by following the quadtree, we propose designing a CNN to quickly determine the final partition for the CTU.
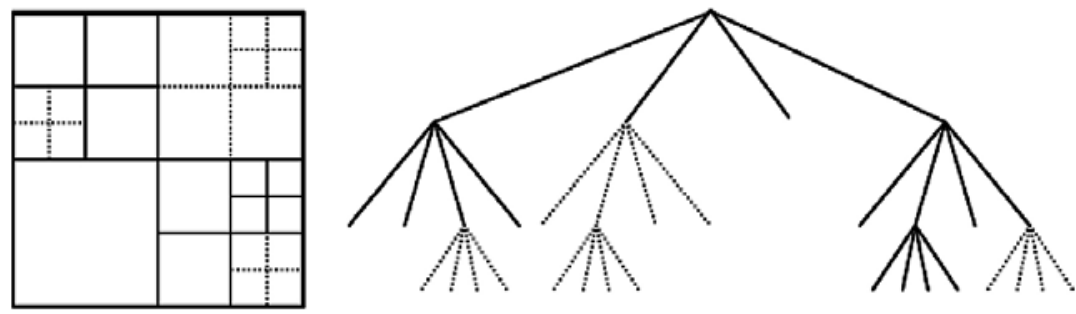

Fig. 2 CU Division Depth Map

\subsection{The Architecture of the Deep NN for Partitioning CTUs}

The architecture of deep NN for partitioning a CTU can be illustrated in Fig. 3. The input is a 64x64 CTU. Each CTU is fed into a CNN with multiple layers. On top of CNN is the full connected layers with Softmax outputting the probability that the input CTU belong to each of partitioning types.

Based on the quadtree, there are totally $174+1=83522$ different possible partitions for a $64 x 64$ CTU. This large number of possibilities leads to the same number of outputs at the Softmax layer, which makes training this deep NN infeasible. One possible solution is that configure each CTU to be the size of 32x32 instead of 64x64. However, this simplified configuration compromises the merit that H.265/HEVC allows larger size of CTU for more efficient encoding. Therefore, our solution is that separating the participating into two steps. The first step uses a deep CNN to determine if the $64 \times 64 \mathrm{CTU}$ needs to be split or not. If so, then the second step is to split the 64x64 CTU into four 32x32 CTUs, and feed each one of them into another deep NN to determine its partitioning mode. Not only the twostep approach is consistent with H.265/HEVC on the maximum size of CTU, but also reduce the number of 32x32 CTUs that need to be fed into the deep NN by the filtering process of the first step.

With respect to the design of the CNN component in the deep NN as shown in Fig. 3, we consider the state-ofthe-art CNN designs, including AlexNet [9], ZF Net [10], VGG [11], GoogleNet [12], and ResNet [13]. We determine that ResNet would be the one that fits our purpose well for the following reasons. First of all, it won ILSVRC 2015 with an incredible error rate of 3.6\% using a revolution of depth of 152 layers; Secondly, it incorporated the effective deep residual learning strategy in its design; Thirdly, all filters that ResNet uses have the 
fixed small size $3 \times 3$, which fit the size of the input CTU (64x64 or 32x32) very well. The depth of ResNet is determined by experimental studies. Since a 64X64 conceptual CTU maps to one 64x64 Luma CTB and two 32x32 Chroma CTB, the real inputs of the deep NN for CTU partitioning should be one 64x64 Luma CTB and two 32x32 Chroma CTB as well. Therefore the final design of the deep NN for CTU partitioning will be as follows:

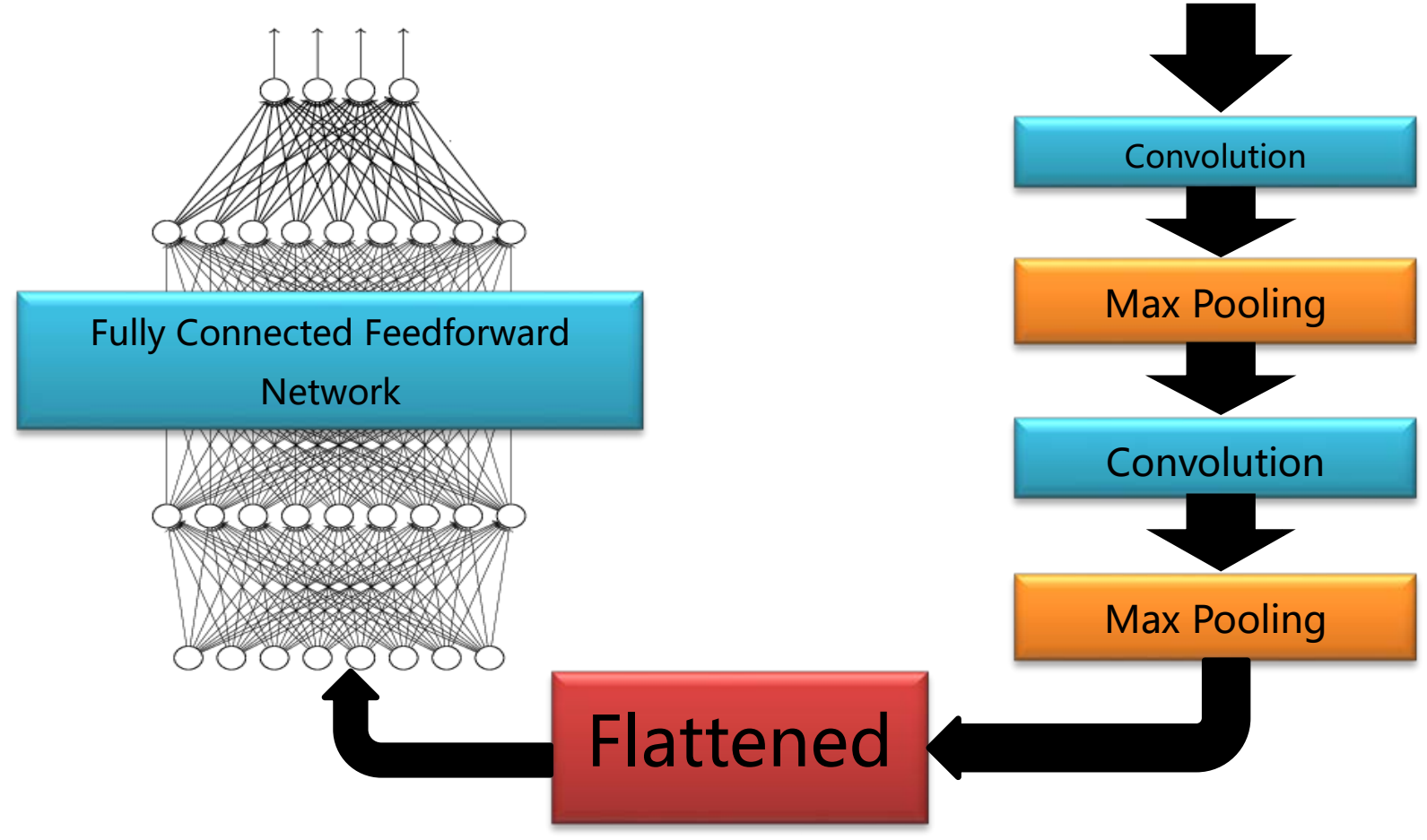

Fig. 3 CNN Architecture

\subsection{Generating Training Data Set}

Generating a large training data set to train the deep NNs described in Section 3.1 is a big challenge. We propose a method that uses H.265 reference software to process a large number of different videos offline in order to generate training data sets. More specifically, we configure the reference software by setting the CTU size to be $64 x 64$, and then output the partition information for each CTU. From the outputs, we extract information to form two training sets. First, for each 64x64 CTU, determine whether it should be partitioned or not. Second, for each 64x64 CTU that is partitioned, extract one of the 17 parturition modes for each of its four 32x32 sub-regions. Using the H.265 reference software to partition CTUs is a time consuming process, which is also one of the primary motivations of our proposal of using deep NN for online CTU partitions. We downloaded 13 videos samples as the training and testing data. Among them, 12 samples were used as training data and the last one is used as testing data. The video samples are in CIF formats with the dimension of 352x288 (as shown in Fig .4). Every frame of the video is divided into CTU with size 32x32. For this sample, the training data includes total of 3150 frames, and each frame has 99 CTUs. So, there are over 300,000 CTUs available for training. The testing data has 260 frames, giving us more than 25,000 CTUs for testing. 


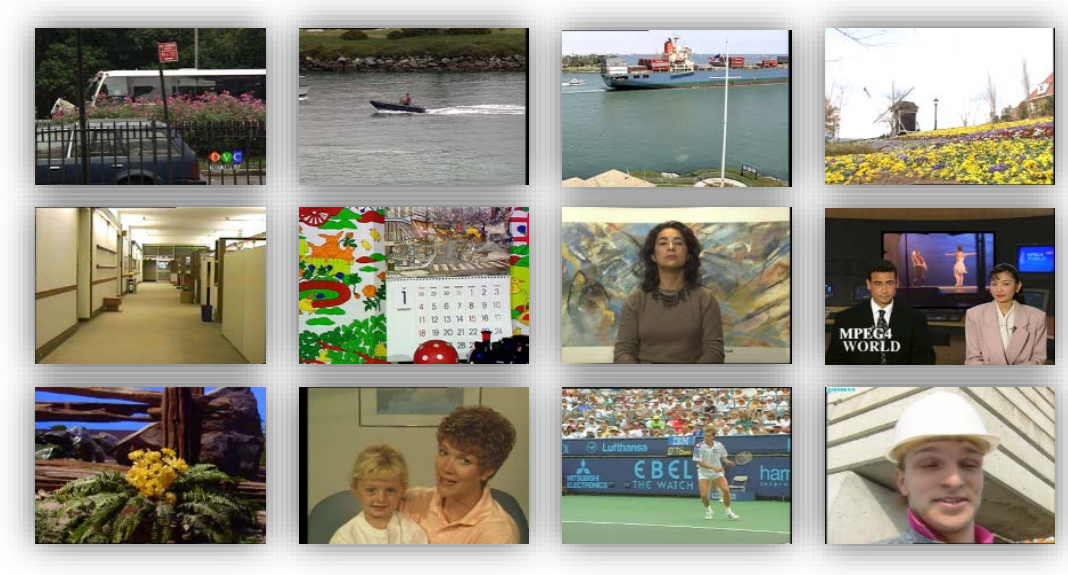

Fig. 4 Video Samples used for Training Set and Testing Set

\subsection{Training Data Set Structure}

For each training sample, we have two components: the input and desired output. The input is basically the gray scale matrix of the 32x32 CTU, and the desired output is the CU depth map of the CTU. Here is how the depth number is defined:

(1) If a $8 \times 8$ block is part of a $32 \times 32 \mathrm{CU}$, then the depth index corresponding to that $8 \times 8$ block is 0 ;

(2) If a $8 \times 8$ block is part of a $16 \times 16 \mathrm{CU}$, then the depth index corresponding to that $8 \times 8$ block is 1 ;

(3) If a $8 \times 8$ block is part of a $8 \times 8 \mathrm{CU}$, then the depth index corresponding to that $8 \times 8$ block is 2 ;

In the following example in Fig. 5, since the 32x32 CTU is divided into $168 \times 8 \mathrm{CUs}$, then the depth index of each $8 \times 8$ block is 2; and thus, every cell in the desired output is 2 .
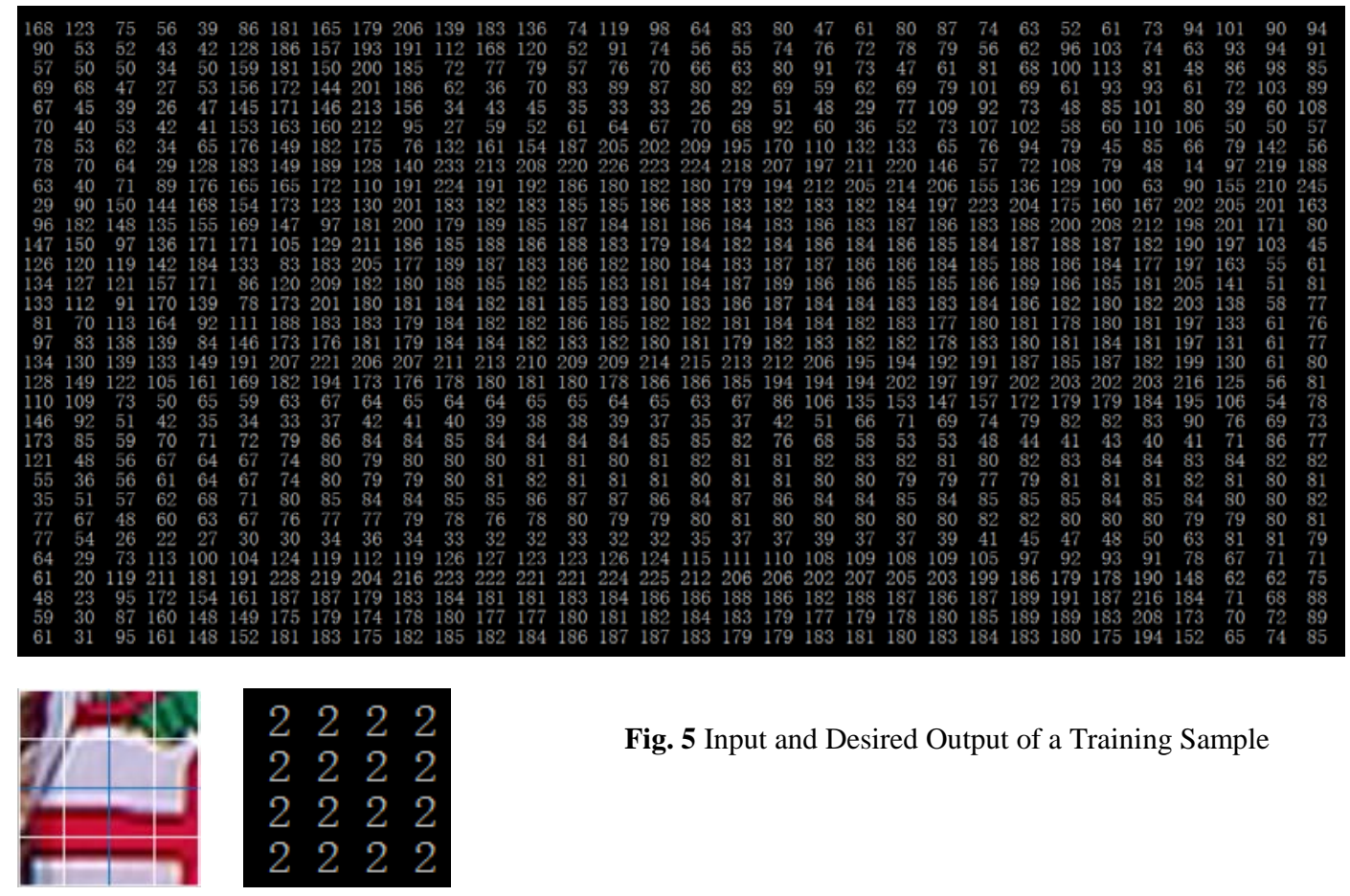

Fig. 5 Input and Desired Output of a Training Sample 


\section{Test Results}

As can be seen from Fig. 6 and Fig. 7, with the CNN we designed, an initial accuracy rate of 60\% was achieved. As the training moves forward, the accuracy rate will increase rapidly and will approach 100\% at around the 200th iteration. In Fig. 6, we have set the value of batch-size parameter to 32, and then we change the value of “Adam”. As can be seen, when the value of "Adam" is too large $(l r=l e-1)$ or too small $(l r=l e-1)$, the results of training are not satisfactory. When we set the value of “Adam" to "lr = le-3", the learning curve will reach $100 \%$ very quickly and will remain stable after that.

In Fig. 7, we have fixed the value of "Adam" to "Ir = le-3", and then change "batch-size" to different values. When the value of "batch-size" is smaller, the accuracy rate will reach $100 \%$ with less number of iterations. However, with smaller "batch-size" value, each iteration will take longer. Thus, we need to choose an appropriate "batch-size" value. Overall, in order to reach satisfactory training results, we need to adjust the values of the parameters. The ultimate goal is to obtain a near-optimum CNN architecture.

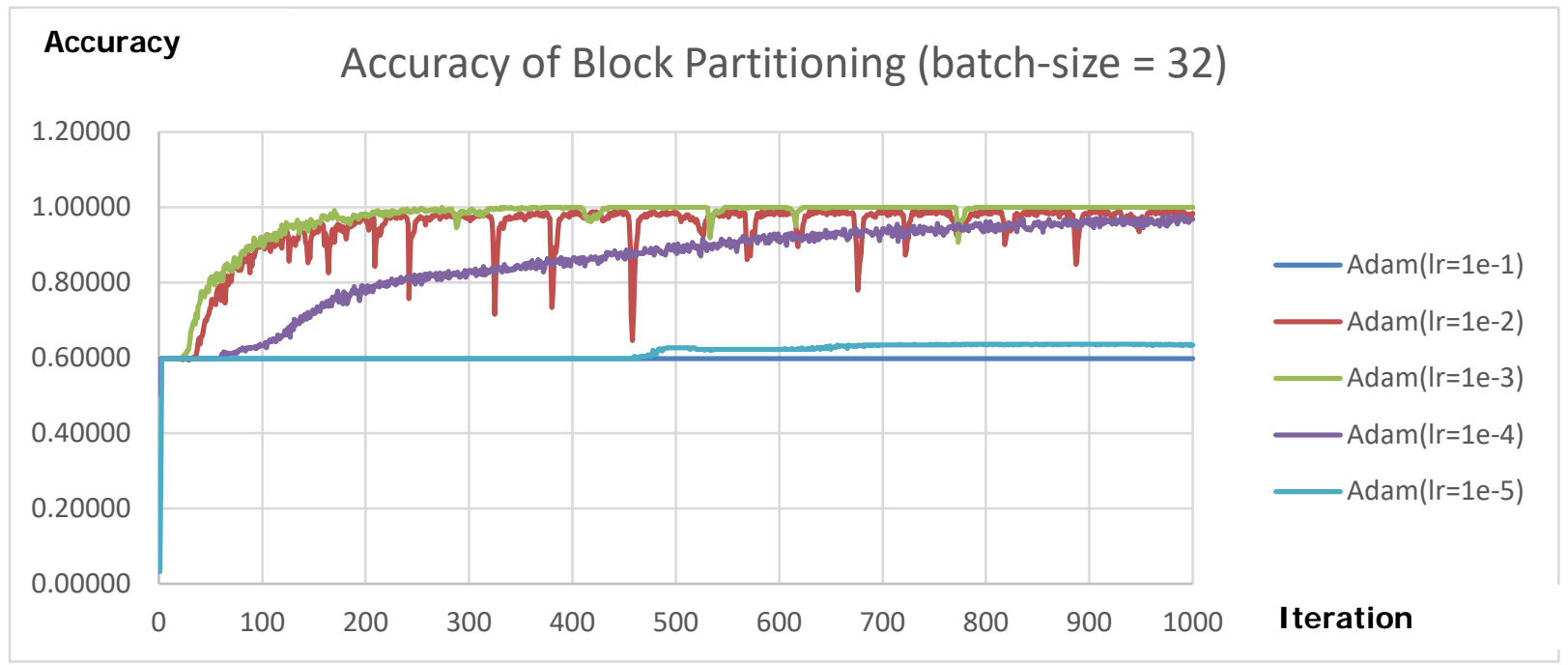

Fig. 6 Accuracy of Blocking (batch-size = 32)

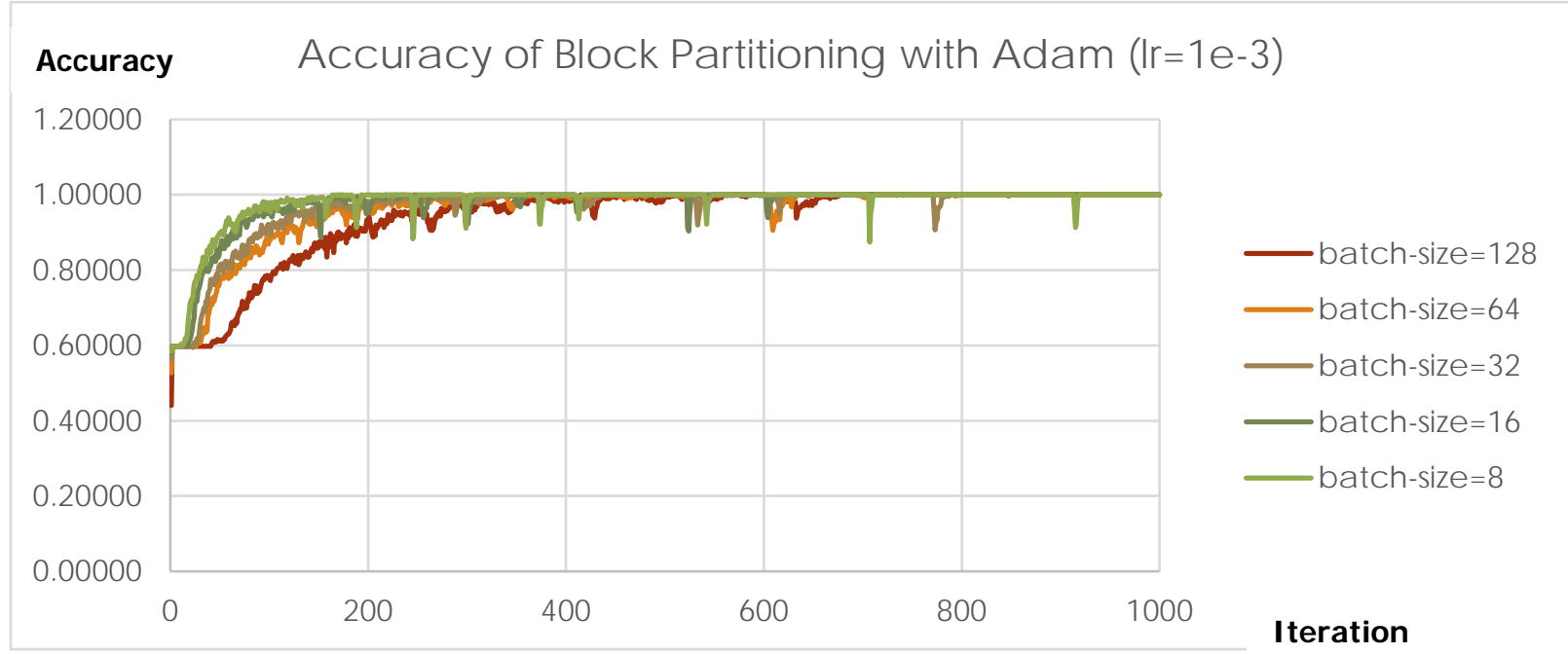

Fig. 7 Accuracy of Blocking with Adam(lr=1e-3) 


\section{Discussion and Future Work}

In this paper, we proposed using deep $\mathrm{NN}$ as the primary technique for efficient video encoding in H.265/HEVC. We proposed several deep NN designs for CTU partitioning in video encoding. One of the biggest challenges for using deep $\mathrm{NN}$ for video encoding is to generate large enough training data for training the designed deep NN models. Our solution to this challenge is using the H.265 reference software to generate the most optimized outputs for each of the above tasks, based on which training data can be extracted. The proposed CNN based algorithm has largely speed up this process (compared to RDO-based block partitioning), and now it can achieve real-time block partitioning. This has enabled the application of H.265/HEVC to real-time application scenarios. Our next step is to conduct large-scale experimental studies on the proposed deep NN designs and refine the designs based on the experimental results. We also plan to apply the proposed algorithm to other modules of H.265/HEVC, such as intra prediction mode decision, intra prediction direction decision, and inter-frame motion estimation.

\section{REFERENCES}

[1] Svetislav Momcilovic, Nuno Roma, Leonel Sousa, Run-time machine learning for H.265/HEVC fast partitioning decision, IEEE International Symposium on Multimedia, 2015, pp347-350

[2] Fanyi Duanmu, Zhan Ma, and Yao Wang, Fast CU partition decision using machine learning for screen content compression, IEEE International Conference on Image Processing (ICIP), 2015, pp4972-4976

[3] Md Mushfiqul Alam, Tuan D. Nguyen, Martin T. Hagan, and Damon M. Chandler, A perceptual quantization strategy for HEVC based on a convolutional neural network trained on natural images, SPIE Applications of Digital Image Processing XXXVIII, Sept. 2015, doi: 10.1117/12.2188913

[4] Zhenyu Liu, Xiaoyu Yu, Yuan Gao, Shaolin Chen, Xiangyang Ji, and Dongsheng Wang, CU partition mode decision for HEVC hardwired intra encoder using convolution neural network, IEEE Transactions on Image Processing, Vol. 25, No. 11, Nov. 2016, pp5088-5103

[5] Zhenyu Liu, Xianyu Yu, Shaolin Chen, Dongsheng Wang, CNN oriented fast HEVC intra CU mode decision, IEEE International Symposium on Circuits and Systems (ISCAS), 2016, pp2270-2273

[6] Zong-Yi Chen, Jiunn-Tsair Fang, Yen-Chun Liu, and Pao-Chi Chang, Machine learning-based fast intra coding unit depth decision for High Efficiency Video Coding, Journal of Information Science and Engineering 32, 2016, pp1289-1299

[7] Gary J. Sullivan; Jens-Rainer Ohm; Woo-Jin Han; Thomas Wiegand, Overview of the high efficiency video coding (HEVC) standard, IEEE Transactions on Circuits and Systems for Video Technology, Vol. 22(12), 2012, pp. 1649-1668.

[8] Jens-Rainer Ohm, Gary J. Sullivan; Heiko Schwarz; Thiow Keng Tan; Thomas Wiegand, Comparison of the coding efficiency of video coding standards - including high efficiency video coding (HEVC), IEEE Transactions on Circuits and Systems for Video Technology, Vol. 22(12), 2012, pp. 1669-1684.

[9] Alex Krizhevsky, Ilya Sutskever, Geoffery E. Hinton, Imagenet classification with deep convolutional neural networks, Advances in Nerual Information Processing Systems (NIPS), 2012

[10] Karen Simonyan, Andrew Zisserman, Very deep convolutional networks for large-scale image recognition, arXiv: 1409.1556, 2014.

[11] Matthew D Zeiler, Rob Fergus, Visualizing and understanding convolutional networks, arXiv:1311.2901, 2013.

[12] Christian Szegedy, Wei Liu, Yangqing Jia, Pierre Sermanet, Scott Reed, Dragomir Anguelov, Dumitru Erhan, Vincent Vanhoucke, Andrew Rabinovich, Going deeper with convolutions, arXiv:1409.4842, 2014

[13] Kaiming He, Xiangyu Zhang, Shaoqing Ren, Jian Sun, Deep residual learning for image recognition, arXiv:1512.03385, 2015 